\title{
Presence of Nicotine in Marketed Nicotine-Free E-liquids for Electronic Cigarettes
}

\author{
Michael $\mathrm{Wu}^{1}$, Helen Heacock ${ }^{2}$, Maria Tirado ${ }^{2}$, Fred Shaw ${ }^{3}$ \\ 1. Lead author, B. Tech student, School of Health Sciences, British Columbia, Institute of Technology, 3700 Willingdon Ave, Burnaby, BC V5G $3 \mathrm{H} 2$ \\ Supervisor, School of Health Sciences, British Columbia Institute of Technology, 3700 Willingdon Ave, Burnaby, BC V5G 3H2 \\ Supervisor, School of Computing and Academic Studies, British Columbia Institute of Technology, 3700 Willingdon Ave, Burnaby, BC V5G 3H2 \\ Contributor, School of Health Sciences, British Columbia Institute of Technology, 3700 Willingdon Ave, Burnaby, BC V5G 3H2
}

\begin{abstract}
Background and Purpose: Ever since the electronic cigarette made its debut in the market, it has been garnering great popularity due to public perception of it being a safer alternative to conventional cigarette. As a result, aside from being utilized in tobacco cessation programs, susceptible populations such as teenagers are slowly adopting this new trend of recreational E-cigarette smoking or "vaping". The literature review conducted suggests that not only do different E-cigarette models exhibit different delivery efficiencies regarding percentage nicotine vapourization, there are discrepancies between what is labelled by the manufacturer and the actual nicotine content in the electronic cigarette liquids. This has serious public health implications because nicotine is the active chemical component in inducing addiction in cigarettes. As a result, recreational electronic cigarette users such as teenagers, may unknowingly become exposed to improper levels of nicotine, leading to a higher probability of nicotine dependence or switching to conventional smoking. The purpose of this study was to determine whether presence of nicotine can be detected in marketed nicotine-free electronic cigarette liquids.
\end{abstract}

Methods: The nicotine content in electronic cigarette liquids was isolated and determined using Gas Chromatography Mass Spectrometry. Descriptive and inferential statistics was conducted using NCSS11 to see if there was a statistically significant difference between the labelled concentration of $0 \mathrm{mg}$ in marketed "nicotinefree" electronic cigarettes from two popular brands, VapeWild and Mt Baker Vapour, to determine whether one brand has better quality control for nicotine content in nicotine-free E-liquids compared to the other brand.

Results: Based on the analyzed E-liquid samples from the two brands, no nicotine was detected.

Conclusion: E-cigarettes can be putatively considered as a safer alternative to conventional cigarettes because nicotine levels can be pre-determined and limited with a high degree of confidence.

Key words: Electronic cigarette, E-liquids, nicotine, accurate labelling, addiction

\section{Introduction:}

Popularity of electronic cigarettes, also known as Ecigarettes, has skyrocketed since they made their debut in the Canadian market in 2007 (1). This "vaping" trend, despite the lack of knowledge regarding its long-term health effects on the users, quickly became mainstream because of manufacturer marketing schemes, claiming it to be a "healthier alternative" to conventional cigarettes. According to Canadian Tobacco, Alcohol, and Drugs Survey (CTADS) in 2013, 9\% of all Canadians ages 15 and older reported having tried E-cigarettes before, which is approximately 2.5 million people (2). In addition, the usage prevalence of E-cigarettes is the highest among young people, with 1 in 5 youth and young adults having tried E-cigarettes before (2). With a $9 \%$ increase from $2007-2013$, the future projections in terms of the number of E-cigarette users can be daunting, especially with the rate being the highest among young people. As this subset of population may be the most susceptible to drug abuse and addiction, the increasing rate of users at an early age can lead to a substantial burden on the current healthcare system and smoking cessation programs. Furthermore, Dr. Tom Frieden theorized that an increase in electronic cigarette uses "[can] reglamorize smoking." (3). 
E-cigarettes are electronic devices that mimic the functionality and appearance of conventional cigarettes. Through the atomizer powered by a battery, E-cigarettes heat a solution (e-juice) composed of various chemicals, including propylene glycol, water, flavouring agents and sometimes nicotine to generate aerosol for inhalation. The major differences compared to conventional cigarettes are that they do not contain tobacco and do not involve the process of combustion (4). The proclaimed "healthier alternative" is partially justified since by circumventing the process of combustion, many of the harmful by-products found in conventional cigarettes are eliminated (4). However, health authorities are concerned that Ecigarettes may serve as a gateway to other tobacco products due to marketing schemes of being "healthier", attractive packaging and most importantly, presence of nicotine. Nicotine has negative impacts on adolescent brain and lung development, making them most susceptible to long-term abuse of E-cigarettes and possibly tobacco cigarettes (5). In addition to nicotine, E-liquids may also contain contaminants that include heavy metals and potential carcinogens such as nitrosamines (6).

At this time, the proposed Federal Tobacco and Vaping Products Act is in the process of amendment, which means the sale and use of this product remains unregulated in Canada (7). E-cigarette products that contain any amount of nicotine falls within the scope of Food and Drugs Act and require approval by Health Canada prior to them being imported, being marketed and sold in Canada. Even though no products have been approved, little enforcement actions have been taken by the Federal regulatory bodies, mostly reacting on a complaint basis (8). As a response to address federal deficiencies, provincial governments have subsequently developed and implemented regulations to mitigate potential health effects of $E$ cigarettes on the users. In British Columbia, the Tobacco and Vapour Products Control Act was introduced, which took effect in September $1^{\text {st }}$ of 2016. It sets out legislative standards for sale, display and promotion of vapour products in stores, the use of vapour products at work places and in public as well as enforcement of the new laws (9). However, there is still much to be studied to confirm that E- cigarettes are in fact a safer alternative to conventional cigarettes. This evidence review investigates potential health risks of E-cigarettes, the discrepancy between the marketing claims of Ecigarettes and the actual products and considers the implications for Federal legislation development, education and enforcement.

This project was inspired by a previous Environmental Health student project on E-cigarette liquids (10), where traces of nicotine in nicotine free liquids were identified. However due to budget constraints, the author was unable to obtain a sufficient sample size to determine whether his findings were statistically significant. In addition, the author did not account for the popularity of brands that he investigated using Gas Chromatography Mass Spectroscopy. Therefore, under BCCDC's sponsorship, the experimental approach was modified and improved in an attempt to find whether or not traces of nicotine are present in marketed nicotine-free E-liquids.

\section{Are E-cigarettes a safer alternative?}

E-cigarettes are being actively marketed as the "safe" new alternative to the conventional cigarettes (11). However, studies are beginning to show that even though by circumventing the combustion reaction, E-cigarettes eliminate the production of numerous carcinogenic by-products that have been characterized in conventional cigarettes, there are still harmful compounds being emitted through this system. In addition to the toxic profile of the vapour produced by E-cigarettes, the public health implications associated should also be considered to determine if the "safer" alternative label is justified.

\section{Carbonyl Compounds}

When the refillable E-liquids incidentally touches the heated nichrome wire, solvent chemicals such as propylene glycol undergo oxidation, forming carbonyl compounds for instance acetaldehyde and formaldehyde. These oxidation products are known to have adverse health effects, which suggest Ecigarettes may not be a safer alternative marketed them to be (12). Uchiyama et al. (2013) measured carbonyl compounds in vapours produced from 13 brands of Japanese E-cigarettes in micrograms per 10 puffs via High Performance Liquid 
Chromatography. Several derivates of carbonyl compounds were investigated; including acetaldehyde, formaldehyde, glyoxal and butanal. Nine of the thirteen brands generated a diverse profile of carbonyl compounds, most notably with high quantities of formaldehyde in aerosols, which is highly toxic (13). The two carbonyl compounds that are specific to E-cigarettes are glyoxal and methylglyoxal, which have shown mutagenicity properties in biological systems, specifically targeting Guanine in DNA. The analysis also revealed large variations in carbonyl concentrations not only among the brands, but across the different samples as well, raising the issue of manufacturing inconsistency or cross-over contamination.

Goniewicz et al. (2014), on the other hand, performed a toxicity profile study on 12 brands of Ecigarettes under controlled conditions and it was found that among the 15 carbonyls investigated, 3 compounds were identified in all the samples: formaldehyde ( 2.0 to $56.1 \mathrm{ug}$ ), acetaldehyde (11.1 to $13.6 \mathrm{ug}$ ), and acrolein (0.7 to $41.9 \mathrm{ug}$ ), all three known to have irritating and toxic properties (12). Kosmider et al. (2014) conducted a similar study to Uchiyama also demonstrated a correlation between variable voltage in second generation EC and levels of carbonyl compounds produced in vapours. It was found that increased battery output voltage produced higher levels of carbonyl compounds in vapour. When voltage was increased from $3.2 \mathrm{~V}$ to $4.8 \mathrm{~V}$, the levels of formaldehyde, acetaldehyde and acetone all demonstrated levels of increase per fifteen puffs, with formaldehyde demonstrating the most significance difference of a 200 fold increase in glycerin/propylene glycol solution. Also, propylene glycol based E-liquids exhibited higher carbonyl levels compared to glycerin-based E-liquids (14). Hutzler et al. (2014) revealed that not only does vaporization efficiency vary across the models, the rate of carbonyl compound production in vapour of E-cigarettes increases after reaching a threshold of puffs. In this puff fraction, which is approximately $40 \%$ of total vapor volume, similar levels of formaldehyde were detected compared to conventional cigarettes (15).

\section{Carcinogens: Tobacco-Specific Nitrosamines}

Tobacco-Specific Nitrosamine (TNSA) is a major chemical component that contributes to the negative health impacts of users. TSNA is a group of potent carcinogenic chemicals derived from cured tobacco leaves and has been found to be at reduced levels in E-liquids compared to conventional cigarettes (16). Farsalinos et al. (2015) compared the levels of TSNA in E-liquid to aerosol generated from E-cigarettes to determine whether heating via nichrome wire contributes to the production of TSNA via UPLC-Mass Spectroscopy. Results show that minimal nitrosamines were found in the three Eliquid samples and TSNAs were detected in all sample aerosols at a level that was not statistically different from the expected. Therefore, pyrolytic aersolization via nichrome wire heating does not contribute to formation of TSNAs (16).

\section{Improper/Mislabelling of Products}

Aside from the toxicants produced in the vapour, product labelling also factors into the safety efficacy of E-cigarettes. False labelling has serious implications on youth addiction and tobacco cessation programs. Buettner-Schmidt et al (2016) conducted a study to investigate the accuracy of the labelled quantity of nicotine content in E-liquids sold in unlicensed vape stores. 70 samples of E-liquids were acquired from 16 unlicensed stores in North Dakota, with a claimed nicotine concentration range of $3 \mathrm{mg} / \mathrm{mL}$ to $24 \mathrm{mg} / \mathrm{mL}$. With a tolerance range of $10 \%$, of the 70 samples, 36 (51\%) were outside of labelled concentration, 24 contained less and 12 contained more than what was labelled. The variation in nicotine concentration ranged from $66 \%$ below to $172 \%$ above what was labelled. In addition, from the 23 samples that claimed to be nicotinefree, 10 (43\%) contained traces of nicotine, from $0.19 \mathrm{mg} / \mathrm{mL}$ to as high as $0.48 \mathrm{mg} / \mathrm{mL}$ (17).

A similar issue was found in the experiment conducted by Hutzler et al. (2014). Of the 10 samples explicitly marketed as nicotine-free, 7 of them were identified containing nicotine in the range of 0.1-15 $\mathrm{ug} / \mathrm{mL}$. The other 18 samples have no indication of nicotine content, which can be mistaken as nicotinefree by the consumer. However, 16 of these samples were found to have nicotine level ranging from 0.1324 ug/mL (16). Davis et al. (2015) also evaluated 
the accuracy of nicotine concentration labelling on Eliquid products by testing nicotine concentration of $71 \mathrm{E}$-cigarette refill fluid products with duplicates, purchased from five different manufacturers. The result showed that out of the 54 that were labelled as containing nicotine, 35 had nicotine concentrations that exceeded the tolerable $+/-10 \%$ range set by AEMSA. The evaluated concentration fluctuated from as little as $1.1 \%$ to as much as $89.7 \%$ from the labelled value, with majority being higher than indicated (18).

\section{E-cigarette Inconsistency}

Nicotine level in refillable liquids is not the only factor that determines efficacy of nicotine delivery into our bodies. Goniewicz et al. (2012) investigated 15 brands, each with only one of the two most popular E-cigarette models (eGo and Dura). It was found that a series of 150 puffs released $0.3-8.7 \mathrm{mg}$ of nicotine in vapours while a series of 300 puffs released $0.5-15.4 \mathrm{mg}$ of nicotine. With a $p$ value of $<0.05$, the difference in aerosolization of nicotine between the different models was found to be statistically significant. On average, $50-60 \%$ of the nicotine in the cartridge is being vaporized for inhalation, which results in overestimation of nicotine uptake (19).

\section{Strengths and Limitations:}

The evidence review collectively demonstrated that health risks associated with E-cigarettes are not just limited to the toxicity profile of the vapours produced, but also the manufacturing consistency of the models and difference in product composition. The interplay of factors is further intensified by the operating parameters conducted by the user, such as voltage control, ultimately resulting in an unregulated product that should be further examined prior to being marketed as a "healthier" alternative. Despite the insights provided on Ecigarettes, this literature review has its limitations. First of all, there is no uniformity in the methodology and data analysis across the different studies, which may contribute to discrepancy between the results found. Also, due to inconsistency in vaporization efficiency across the brands and models, underlying systematic errors may contribute to the amount of nicotine and toxic compounds measured in the experiments. While most literature focuses on the toxicity profile of the vapour, flavouring additives and carrier solvents should also be explored in further detail to consider their health implications, as they might be potential allergens and irritants.

Most refillable liquids and E-cigarettes tested are the big and most popular brands, but smaller local brands should also be considered since there is a high chance of poor quality control and technical issues. Last but not least, the studies are carried out under different parameters, including voltage operation of the E-cigarettes. Therefore, results from different experiments cannot always be compared.

\section{Implications:}

Goniewicz et al (2012) and Buettner-Schmidt et al (2016) revealed the inconsistency in the manufacturing process for both the refillable liquids and the E-cigarette models among all the brands. Mislabelling or absence of labelling of E-liquid composition, as discovered in Buettner-Schmidt et al (2016) study, is a significant issue regarding nicotine content since the majority of the refillable liquids have been found to have failed the acceptable level of $+/-10 \%$ of the labelled value, regardless of the brand. Nicotine is both addictive and toxic and if utilized in tobacco cessation programs, the addicts will not be prescribed with the appropriate dosage of nicotine if these E-liquids are used, disrupting their cessation progress. Sudden increases or decreases in nicotine intake can result in a deviation from the treatment regime leading to withdrawal or overdose symptoms and possibly induce compulsory smoking.

The lack of quality control and consistency in these products is mainly attributable to the absence of legislation and the regulatory body that conducts such enforcements to protect the public. Therefore, a definition for E-cigarette needs to be added into the Federal Tobacco Act and Tobacco Enforcement Officers need to be educated and trained regarding distribution of sale for minors and proper advertising. Also a standardized testing plan should be required by all E-cigarette manufacturers and it should be approved before receiving a business permit. The Kosmider et al. (2014) study demonstrated two important variables affecting carbonyl production in E-cigarettes: variable voltage control and type of carrier solvent. It has been found 
that the higher voltage used for E-liquid vaporization, the more carbonyl compounds are generated. Therefore, regulations should be devised and implemented to require disclaimers to be included in product usage instructions with respect to proper operating voltage level, difference between different types of carrier solvents and the health implications behind them.

\section{Methods and Materials}

\section{Description of Materials used}

The method used to detect and identify nicotine levels in marketed "nicotine-free" liquids is Gas Chromatography coupled with Mass Spectrometry (GC-MS), which is an analytical method capable of separating and identifying complex mixture of chemicals.

\section{GC-MS Standard Procedure}

\section{Theory of GC-MS}

The theory behind this technique is that different components within a mixture such as E-cigarette liquids interact with the stationary phase with various affinity. The stronger the interaction, the tighter the component adsorbs to the stationary phase, the longer it takes for it to pass through the column via mobile phase (20). Before analysis can begin, the samples must be prepared by extracting analyte of interest into a solvent phase. The samples are then injected into the sample port of GC, where they are volatilized and swept through the column by an inert gas phase such as helium. Carried by the inert carrier gas, the different components of the samples are separated based on the affinity of interaction with the stationary phase in the column (26). Compounds that have low affinity with the stationary phase travel through the column rapidly, yielding a shorter retention time as opposed to those with higher affinity that travel more slowly, yielding a longer retention time.

Once the individual analytes exit the GC column, they enter the ionization area of Mass Spectrometry, where they are bombarded with electrons to form ionized fragments of the analyte. They are then accelerated through a magnetic field generated by direct current and voltage and depending on their mass and ionic charge, the degree of curvature of the paths the fragments travel differ prior to reaching the detector, creating a distinct mass-tocharge profile for each component of the samples. Finally, the MS computer detects and graphs a mass spectrum scan showing the relative abundance of each ionized mass fragments at corresponding retention time (21). Refer to Appendices $A 1$ for the schematics of GC-MS set-up.

\section{GC-MS Mode}

Two modes in GC-MS were used to detect presence of nicotine in the samples

Full Scan mode - used for identification of chemical components using a mass spectrum. In this mode, GC-MS monitors a range of masses generated by bombardment of electrons. The resulting spectra is compared to computer libraries containing mass spectra of many different compounds to identify unknown analytes in a sample (22).

SIM mode - used for quantitative analysis of trace components when the mass spectra of the trace components are known. In SIM mode, GC-MS gathers masses specific to the analyte of interest vs looking for all masses in a specified range. Typically two to four ions unique to the analyte of interest are monitored (22).

\section{Methodology}

Based on accessibility in Canada and popularity vote on a public E-cigarette forum, 30 samples of E-liquids from two brands, Vape Wild and Mt Baker Vapour, were purchased directly from the manufacturers. Gas Chromatography Mass Spectrometry was used to analyze the 60 samples for presence of nicotine. Based on this standard procedure, the Limit of Detection (LoD) was determined to be 0.013 $\mu \mathrm{g} /$ sample. The precision, as determined from the pooled relative standard deviation ( $\mathrm{Sr}$ ) was 0.024 (23). Low LoD and Sr values show that this analytical method is suitable for intended purpose since it is highly sensitive to analyte of interest and is consistent in measuring minuscule levels of nicotine between the samples (24). 


\section{GC-MS Analysis}

A measured amount of 0.050 grams of nicotine was dissolved in $50 \mathrm{~mL}$ methanol in a volumetric flask to make a $1000 \mathrm{mg} / \mathrm{L}$ concentrated nicotine standard solution. The same process was performed for quinoline to make a $1000 \mathrm{mg} / \mathrm{L}$ internal standard solution(25).

Various volumes of nicotine stock solution were pipetted out and diluted with methanol to make 10ppm, 25ppm, 50ppm, 75ppm and 100ppm nicotine solutions as standards with the intention of generating a 5-point calibration curve for quantification if nicotine was found (20). $0.5 \mathrm{~mL}$ of quinoline was added equally to each standard solution. To confirm the proper retention time of nicotine and quinoline under determined parameters, $1 \mathrm{ml}$ of $100 \mathrm{ppm}$ standard solution was transferred into $1 \mathrm{~mL}$ vial for GC-MS analysis.

Samples were left to run overnight under scan mode without any preparation. The parameters were previously determined by supervisor for optimal readings. Data was collected and chromatogram of each sample was integrated into GC-MS library to identify potential presence of nicotine (26). Results were reconfirmed by running the same samples overnight using SIM mode.

\section{Inclusion and Exclusion Criteria}

The author tested only the E-liquids that claim to be nicotine-free or have 0 nicotine levels shown on product labels. Aside from that, there are no inclusion/exclusion criteria, since there is no prior knowledge regarding the different manufacturers, flavours or brands. The samples were chosen based on popularity from the public and lower cost as determined from internet forums.

\section{Ethical Consideration}

There is no ethical consideration for this study because it does not involve conscientious test subjects. Therefore the boundaries of autonomy and beneficence are not violated.

\section{Results}

The nicotine analysis of E-liquids from two popular brands, Vape Wild and Mt Baker Vape, using GC-MS are summarized in tables 1 and 2. Nicotine was not detected in all 60 samples purchased directly from the manufacturers in the United States. Using the GC-MS scan mode, some of the major chemical species identified were glycerin, which is the part of the matrix and ethyl vanillin, which is a flavouring agent. However, the expected peak of nicotine at 4.035 min acqusition time was not found. Thus, all samples tested negative for the presence of nicotine in the E-liquids.

\begin{tabular}{|c|c|c|c|c|c|}
\hline $\begin{array}{l}\text { Vape Wild - } \\
\text { Fruit Hoops } \\
\text { (Sample set A) }\end{array}$ & $\begin{array}{l}\text { Peak at } \\
4.035 \\
\text { minutes? }\end{array}$ & $\begin{array}{l}\text { Presence/Ab } \\
\text { sence of } \\
\text { Nicotine } \\
\text { (below LoD) }\end{array}$ & $\begin{array}{l}\text { Mt Baker Vapor } \\
\text { - Fruity Hoops } \\
\text { (Sample Set B) }\end{array}$ & $\begin{array}{l}\text { Peak at } \\
4.035 \\
\text { minutes? }\end{array}$ & $\begin{array}{l}\text { Presence/Absence } \\
\text { of Nicotine }\end{array}$ \\
\hline A1 & No & Absence & B1 & No & Absence \\
\hline$A 2$ & No & Absence & B2 & No & Absence \\
\hline A3 & No & Absence & B3 & No & Absence \\
\hline A4 & No & Absence & B4 & No & Absence \\
\hline A5 & No & Absence & B5 & No & Absence \\
\hline A6 & No & Absence & B6 & No & Absence \\
\hline A7 & No & Absence & B7 & No & Absence \\
\hline A8 & No & Absence & B8 & No & Absence \\
\hline A9 & No & Absence & B9 & No & Absence \\
\hline
\end{tabular}




\begin{tabular}{|l|l|l|l|l|l|}
\hline A10 & No & Absence & B10 & No & Absence \\
\hline A11 & No & Absence & B11 & No & Absence \\
\hline A12 & No & Absence & B12 & No & Absence \\
\hline A13 & No & Absence & B13 & No & Absence \\
\hline A14 & No & Absence & B14 & No & Absence \\
\hline A15 & No & Absence & B15 & No & Absence \\
\hline A16 & No & Absence & B16 & No & Absence \\
\hline A17 & No & Absence & B17 & No & Absence \\
\hline A18 & No & Absence & B18 & No & Absence \\
\hline A19 & No & Absence & B19 & No & Absence \\
\hline A20 & No & Absence & B20 & No & Absence \\
\hline A21 & No & Absence & B21 & No & Absence \\
\hline A22 & No & Absence & B22 & No & Absence \\
\hline A23 & No & Absence & B23 & No & Absence \\
\hline A24 & No & Absence & B24 & No & Absence \\
\hline A25 & No & Absence & B25 & No & Absence \\
\hline A26 & No & Absence & B26 & No & Absence \\
\hline A27 & No & Absence & B27 & No & Absence \\
\hline A28 & No & Absence & B28 & No & Absence \\
\hline A29 & No & Absence & B29 & No & Absence \\
\hline A30 & No & Absence & B30 & No & Absence \\
\hline
\end{tabular}

\section{Result Interpretation:}

Based on the ionic fragments detected and comparison with the existing mass spectral library, the broad peak observed ranging from $3.25 \mathrm{~min}$ to $4.4 \mathrm{~min}$ acquisition time, as shown in figure 1 , was contributed by glycerin. The small peak observed around 4.5 min acquisition time was contributed by ethyl vanillin and the multiple aggregated peaks after $5.3 \mathrm{~min}$ acquisition time was predominantly contributed by ethyl citrate.

The rationale behind concluding that there was nicotine in the samples was because of the

steady increase of the slope of glycerin peak that overlapped with the expected peak of nicotine at $4.035 \mathrm{~min}$, as shown in figure 1 . The $\mathrm{Y}$-axis measures the relative abundance of the ionic species found at the corresponding retention time. If, in addition to glycerin, nicotine was present, a peak should have been evident at $4.035 \mathrm{~min}$ rather than a flat slope, since there were two species contributing to the abundance of ionic fragments rather than just glycerin. From figure 2, the two predominant ionic fragments detected at 4.035 min were 43.1 and 61.1. 


Fragmentor
Voltage $\quad \begin{gathered}\text { Collision } \\ \text { Energy }\end{gathered} \quad 0 \quad \begin{gathered}\text { Ionization } \\ \text { Mode }\end{gathered} \quad \begin{gathered}\text { Unspecifie } \\ d\end{gathered}$

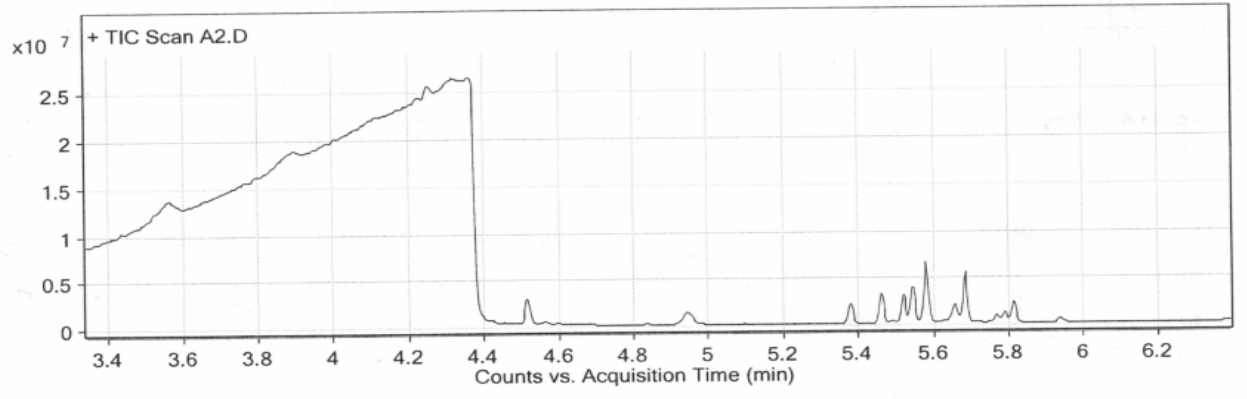

Figure 1: Sample A2 Chromtogram generated using GC-MS Scan mode

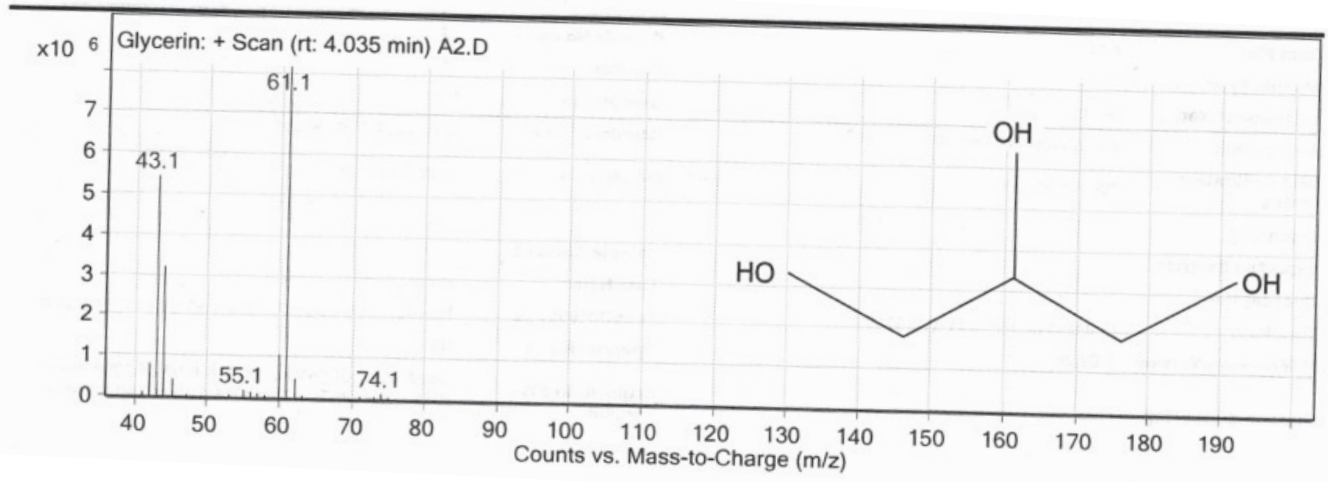

Figure 2: Sample A2 lonic fragments generated at $4.035 \mathrm{~min}$ acquisition time

The SIM mode was also conducted to confirm the potential presence of nicotine by detecting ionic fragments specific to the analyte of interest. Based on the nicotine standard chromatogram shown in figure 4, the two ionic fragments that characterize nicotine are 84.2 and 133.1. However, multiple peaks with the same two ionic fragments generated by nicotine were shown at different retention times, none of which corresponded to the expected acquisition time of $4.035 \mathrm{~min}$, as shown in the figure 3 . This suggested that there might be other chemical species in the E-liquids that generate the same ionic fragments as nicotine. All 60 samples displayed similar patterns in their chromatogram. Therefore, the null hypothesis cannot be rejected and no nicotine was present in the samples. 


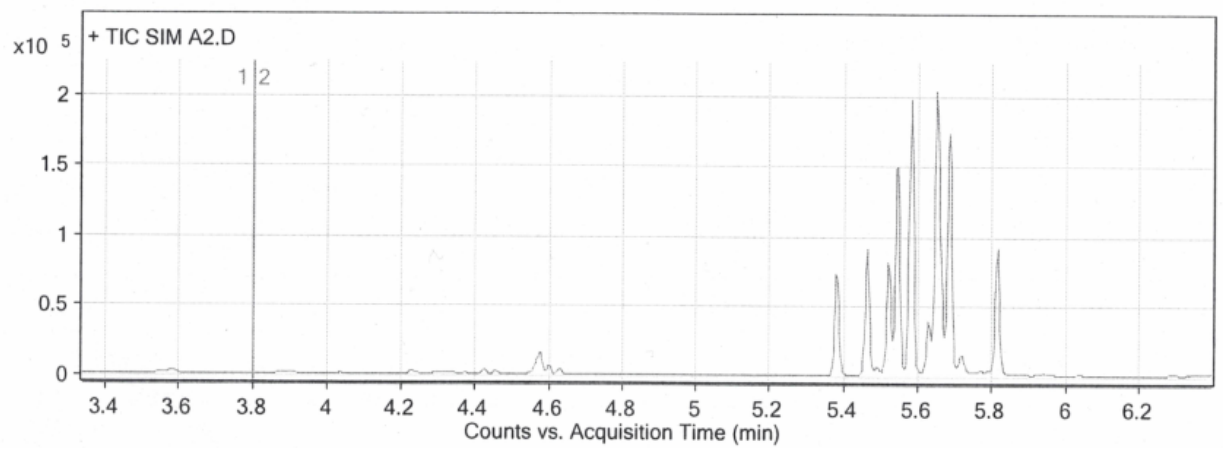

Figure 3: Sample A2 Chromtogram generated using GC-MS SIM mode

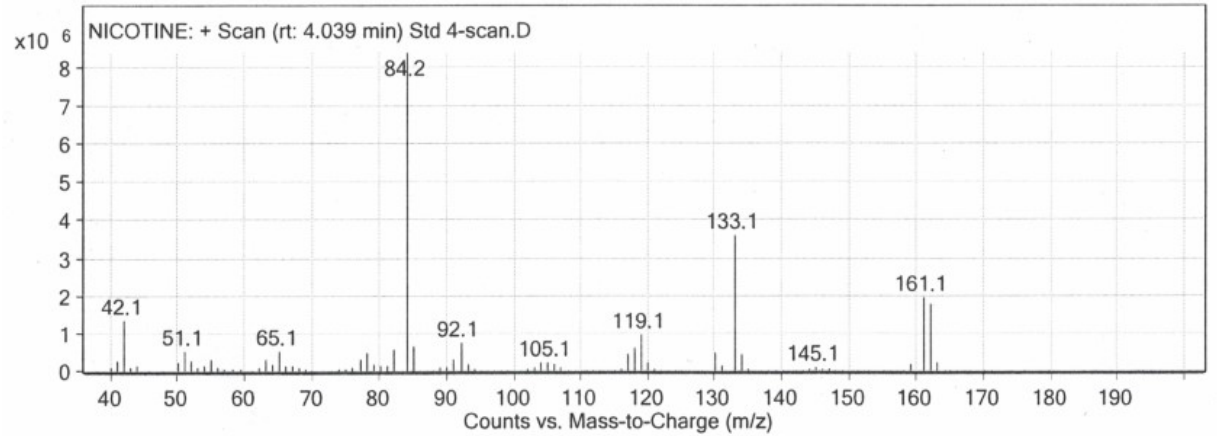

Figure 4: Chromatogram of standard 4 with only nicotine

\section{Discussion}

The efficacy of E-cigarettes for recreational and tobacco cessation purposes has been highly controversial among consumers and healthcare professionals since they became commercially available for the general public. The absence of nicotine in all samples from the two electronic liquid brands, Mount Baker Vapor and Vape Wild, is a vindicating finding for both the electronic cigarette users and the manufacturers. This suggests the composition of E-liquid products from the two popular brands is in accordance with the product label as $0 \mathrm{mg}$ of nicotine or "nicotine-free" and quality control strategies have been implemented in the manufacturing process to mitigate cross-contamination between batches of various nicotine concentrations. Therefore, the public health significance from potential nicotine exposure for E-cigarette users using these two brands is minimal. To extrapolate the results further, E-cigarettes can be putatively considered as a safer alternative to conventional cigarettes because nicotine levels can be pre-determined and limited with a high degree of confidence.

Gas Chromatography Mass Spectroscopy is a robust and highly sensitive analytical method for separation and detection of multicomponent mixtures. But due to the imperfect nature of analytical techniques, it is restricted by the limit of detection (LOD), which is defined as the lowest concentration obtained from the measurement of a sample that can be differentiated from a blank (26). As previously discussed, the LoD is $0.013 \mu \mathrm{g}$ per sample. In comparison with a human LD50 of $0.8 \mathrm{mg} / \mathrm{kg}$ extrapolated from animal studies (27), any amount of nicotine under $0.013 \mu \mathrm{g}$ has minimal public health implication, which is why the samples can be presumed as "nicotine-free".

Public skepticism towards the emerging ecigarette culture remains evident despite the continuous portrayal and repackaging of the product as a healthier alternative to conventional cigarettes. This stems primarily 
from the lack of studies addressing the chronic effects that E-cigarettes may potentially pose on human body. Also, E-cigarette has yet to be defined under any federal legislation $(28,29)$, which generates many loopholes and contradicting information around the sale and usage of the product. These reasons emphasize the importance of scientific investigation, which allows the public to segregate the health evidence from the false messages. Realizing the deficiencies exist in the E-cigarette industry, Bill S- 5 was introduced in the senate on November $22^{\text {nd }}, 2016$ for the amendment of the Tobacco Act and Non-smokers' Health Act to Tobacco and Vaping Products Act (30). The negative results for nicotine in this research are inconsistent with previous studies conducted by Buettner-Schmidt et al (17) and Hutzler et al (15), where they discovered discrepancy with product labels and actual nicotine levels found in the marketed "nicotine-free" E-liquids. The contrasting scientific evidence suggests additional experiments are required to determine which brands are in compliance with quality control. At this point in time, the negative results suggest that Vape Wild and Mount Baker Vapour E-liquids have minimal public health implications regarding youth addiction and the nicotine levels are either absent or far below safety standards to result in adverse health effects.

\section{Limitations:}

The 30 samples from each brand were ordered all at once, which suggests that they were manufactured in the same batch. Therefore, the samples may not be representative of the manufacturer's consistency of quality control and operational standards. Instead, it provides more of a snap-shot of whether there was cross-contamination of nicotine limited to this particular batch. Purchasing a portion of the samples at different time points throughout the course of the study can potentially yield a more accurate representation of the manufacturing operation over a specific time frame.

Another limiting factor is that GC-MS, although highly sensitive, is not $100 \%$ specific for analyte of interest. There can be other chemical compounds that interact with the column with a similar affinity, which can result in interfering signals. One feasible solution to eliminating interfering chemical species is to isolate nicotine by solvent extraction so a majority of the matrix can be removed to enhance desired signal.

Due to time constraint and external factors that were unexpected, any subsequent troubleshooting or adjustments to the experiment could not be performed, including supervisor's proposed idea of diluting the samples to minimize the signals contributed by the matrix.

\section{Knowledge Translation}

Although the results indicated no public health implications with nicotine in marketed "nicotine-free" E-liquids from Mount Baker Vapor and Vape Wild, more studies are required to address the inconsistency in quality across the industry. But the results from this study support that E-cigarettes can be adopted in tobacco cessation programs because nicotine can be controlled to the proper concentration with a high degree of labelling reliability.

To encourage higher quality E-liquids produced by the manufacturers and to allow consumers to make well-informed decisions about what they choose to smoke, a national standard certification committee for E-liquids should be created, where manufacturers can be audited based on a set of criteria, including labelling accuracy and emission level of toxicants, to ensure public health safety associated with the use of these products. Once the products are qualified, they will be branded with a label of recognition, allowing the public to know that the quality of the products is guaranteed by a professional certification body. Legislation governing labelling of products is prescribed under Food and Drugs Act (29). Although GC-MS was unable to detect any presence of nicotine for the two brands of $\mathrm{E}$ - 
liquids, Mt Baker Vapor and Vape Wild, generalization cannot be made about all the brands in the market. Standard of operation and quality control strategies vary between manufacturers, which can lead to products of various qualities and chemical composition. To prevent ambiguity of the legislation, a section specifying a maximum allowable discrepancy range between labelled and actual nicotine concentration in E-liquids should be included under the Food and Drugs Act. This gives the manufacturers the responsibility to maintain high levels of quality control and self-regulate their operations.

\section{Conclusion:}

The findings of this research cannot reject the null hypothesis that there is no inconsistency with the labelled nicotine level in marketed "nicotine-free" E-liquids and the actual nicotine concentration in the samples. Therefore, public health implications regarding nicotine exposure

accurate advice to the public and utilize Ecigarettes in a beneficial and safe manner.

\section{Future Research:}

1. Buettner-Schmidt et al determined that of the 70 samples tested, $51 \%$ of the labels of E-cigarette liquids do not accurately reflect the levels of nicotine found in the products. In addition, out of the $23 \mathrm{E}$-liquids that claimed to be "nicotine-free", $43 \%$ contained nicotine. These findings were not consistent with the results found in this study, which raises the question of whether or not the brands used in Buetnner-Schmidt's study were from smaller local manufacturers. Since there are currently no Federal legislation associated with vaping, the loophole permits any individual to produce their own E-liquids at their own liberty with no legal abidance to quality or labelling standards. Therefore, future studies can with the use of "nicotine-free" liquids is minimal. This is under the assumption that the users purchase the E-liquids from the two brands that were tested in this study. Generalizations cannot be made about all Eliquids in the market, because federal legislation regarding vapour products is still underdeveloped, giving any individual the power to synthesize their own formulation of $\mathrm{E}$ liquids without commercial restrictions, which often entails cross-contamination of products and chemicals. Without any safeguards to the quality of the E-liquids, recreational users can unwittingly become exposed to nicotine, leading to nicotine addiction. The results of this study contradicts some studies currently posited by the scientific community, which is why it is crucial to continue E-liquid testing on domestic and international brands for chemical composition and aerosol profile. Only by gaining a more holistic view regarding vapour products in the market can the health professionals provide accurate and helpful recommendations to the general public.

focus on smaller local brands in Canada that have a higher risk of crosscontamination and non-compliance with the labelling requirements.

2. Another aspect of E-cigarette that has not been extensively investigated is the nichrome heating unit in replacement of combustion. This has been the main premise that sustains the claim that Ecigarette is a healthier alternative because it by-passes the process of combustion in conventional cigarettes. However, Hess et al (31) in their study found high levels of toxic metals, including chromium, lead and nickel in all five leading brands. The sources of the metal have been putatively associated with the nichrome heating unit that comes in contact with the liquids. Future students can confirm the findings by doing a chemical composition analysis of E-liquids before and after contacting the nichrome 
wiring to determine the causality of heavy metals in the E-liquids and perhaps in conjunction with an engineer, determine a more effective volatilization design for E-cigarettes.

3. Surveys can be conducted targeting young adolescents in high schools to investigate the general opinion about the vaping culture and what factors can potentially instigate them to start vaping. By using an open-ended style survey, this study can potentially reveal the underlying causality behind $\mathrm{E}$ cigarette addiction and devise optimal strategies for E-cigarette reduction.

\section{References}

1. Ripton, N (2016). Are electronic cigarettes safe? Canadian Living. Retrieved from: http://www.canadianliving.com/health/ prevention-and-recovery/article/areelectronic-cigarettes-safe

2. Czoli CD, Reid JL, Rynard VL, Hammond D (2015). E-cigarettes in Canada Tobacco Use in Canada: Patterns and Trends. Waterloo, ON: Propel Centre for Population Health Impact, University of Waterloo. Retrieved from: http://www.tobaccoreport.ca/2015/To baccoUseinCanada 2015 EcigaretteSup plement.pdf

3. Partnership for Drug Free Kids (2014). Ecigarettes are as dangerous as regular cigarettes: CDC Director. Retrieved from:

http://www.drugfree.org/jointogether/ ecigarettes-are-as-dangerousasregularcigarettes-cdc-director/

4. Belluz, Julia (2015). E-cigarettes and health - here's what the evidence

\section{Acknowledgement}

I would like to express my deepest gratitude to my research supervisor, Helen Heacock, for her ongoing support and constructive feedbacks throughout the duration of my project. Acknowledgement is also warranted for Dr. Maria Tirado, for her expertise in GC-MS and analytical chemistry as well as the rest of the chemistry department for sharing the laboratory workspace. I am extremely lucky to have her as a mentor and have access to all the resources required for my project. Lastly, I would like to show my appreciation toward Lorraine Mclntyre and BCCDC for sponsoring my research project. It would not have been possible without their financial support

actually says. Retrieved from

http://www.vox.com/2015/6/26/88323

37/E-cigarette-health-fda-smokingsafety

5. CDC (2015). Health Effects of Cigarette Smoking. Retrieved from:

https://www.cdc.gov/tobacco/data_sta tistics/fact_sheets/health_effects/effect s_cig_smoking/

6. Cheng T. (2014). Chemical evaluation of electronic cigarettes. Tobacco Control. ;23:ii11-ii17. Retrieved from: http://dx.doi.org/10.1136/tobaccocontr ol-2013-051482.

7. Health Canada. (2016). Government of Canada Introduces New Tobacco and Vaping Products Legislation. Retrieved from: http://news.gc.ca/web/articleen.do?nid=1158489

8. MetroNews. (2014). E-cigarette with nicotine illegal, but shops still selling product. Retrieved from: http://www.metronews.ca/news/ottaw a/2014/07/23/E-cigarettes-withnicotine-illegal-but-shops-still-sellingproduct.html 
9. Tobacco and Vapour Products Control Act (R.S.B.C. 1996, c. 451). March 22 ${ }^{\text {nd }}$, 2017. Queen's Printer, Country of Canada. Retrieved from: http://www.bclaws.ca/civix/document/i d/complete/statreg/96451_01

10. Sham, J., Karakilic, V., Soulsbury, K., Shaw, F. (2015). Electronic Cigarettes: Using Gas Chromatography Mass Spectrometry Analysis on Nicotine Free Electronic Cigarette Liquids. Personal Collection of V. Karakilic, British Columbia of Institute and Technology, Burnaby BC

11. Bach, Laura (2015). The Path to Tobacco Addiction Starts at Very Young Age. Retrieved from https://www.tobaccofreekids.org/resea rc h/factsheets/pdf/0127.pdf

12. Goniewicz, M., Knysak J., Gawron M., Kosmider L., Sobczk A., Kurek J., Prokopowicz A., Jablonska-Czapla M., Rosik-Dulewska C., Havel C., Jacob P., Benowitz N. (2014). Levels of selected carcinogens and toxicants in vapour from electronic cigarettes. Tobacco Control, 23(2).

13. Uchiyama S., Bekki K., Ohta K., Inaba Y., Nakagome H., Kunugita N. (2014). Carbonyl Compounds Generated from Electronic Cigarettes. Int. J Environ Res Public Health.

14. Kosmider, L., Sobczak, A., Fik, M., Knysak, J., Zaciera, M., Kurek, J., \& Goniewicz, M. L. (2014). Carbonyl compounds in electronic cigarette vapors-effects of nicotine solvent and battery output voltage. Nicotine \& Tobacco Research.

15. Hutzler, C., Paschke, M., Kruschinski, S., Henkler, F., Hahn, J., \& Luch, A. (2014). Chemical hazards present in liquids and vapors of electronic cigarettes. Archives of toxicology, 88(7), 1295-1308.
16. Farsalinos, K. E., Gillman, I., Melvin, M. S., Paolantonio, A. R., Gardow, W. J., Humphries, K. E., Brown, S. E., Poulas K., \& Voudris, V. (2015). Nicotine Levels and Presence of Selected TobaccoDerived Toxins in Tobacco Flavoured Electronic Cigarette Refill Liquids. Int. J Environ Res Public Health, 12(4), 34393452.

17. Buettner-Schmidt, K., Miller, D.R., Balasubramanian, N., (2016). Electronic Cigarette Refill Liquids: ChildResistant Packaging, Nicotine Content, and Sales to Minors. J Pediatr Nurs. 31(4): 373-379.

18. Davis, B., Dang, M., Kim, J., Talbot, P. (2015). Nicotine Concentrations in Electronic Cigarette Refill and Do-ItYourself Fluids. Oxford Journals Nicotine and Tobacco Research. Nicotine Tob Res. 17(2): 134-141.

19. Goniewicz, M., Kuma T., Gawron M., Knysak J., Kosmider L. (2012). Nicotine Levels in Electronic Cigarettes. Nicotine \& Tobacco Research. Nicotine Tob Res. 158-66. doi: 10.1093/ntr/nts103.

20. Amersham Biosciences UK Ltd. Reverse Phase Chromatograph [Internet]. Amersham UK Ltd. 1999 [cited 2016 Nov 20]. Available from: http://wolfson.huil.ac.il/purification/PD F/ReversePhase/AmershamRPCManual. pdf.

21. Gates, P. (2017). Gas Chromatography Mass Spectrometry (GC/MS). [Internet]. [cited 2017 Feb 24]. Available from: http://www.bris.ac.uk/nerclsmsf/techni ques/gcms.html

22. ALS Environmental. Lab Science News Technical Blog: GC/MS - Full Scan vs GC/MS - SIM. [Internet]. [cited 2017 Feb 24]. Available from: http://www.caslab.com/News/gcmsfull-scan-vs-cgms-sim.html 
23. Pendergrass M, Jaycox L. Nicotine. NIOSH Manual of Analytical Methods. Fourth Edition [Internet]. [cited 2016 Nov 20]. Available from:

https://www.cdc.gov/niosh/docs/2003154/pdfs/2551.pdf

24. Pendergrass M, Jaycox L. Nicotine. NIOSH Manual of Analytical Methods. Fourth Edition [Internet]. [cited 2016 Nov 20]. Available from: https://www.cdc.gov/niosh/docs/2003154/pdfs/2551.pdf

25. Sham, J., Karakilic, V., Soulsbury, K., Shaw, F. (2015). Electronic Cigarettes: Using Gas Chromatography Mass Spectrometry Analysis on Nicotine Free Electronic Cigarette Liquids. Personal Collection of V. Karakilic, British Columbia of Institute and Technology, Burnaby BC

26. Heyden, Y.V., Boque, R. (2009). The Limit of Detection. LC/GC Europe. Retrieved from: http://www.chromatographyonline.co m/limit-detection

27. Mayer, B. (2013). How much nicotine kills a human? Tracing back the generally accepted lethal dose to dubious self-experiments in the nineteenth century. Arch Toxicol. 2014; 88(1): 5-7.
28. Canadian Press. (2016). Liberals Introduce New Vaping Legislation. Huffingtonpost. Retrieved from: http://www.huffingtonpost.ca/2016/11 L22/liberal-government-introducesnew-tobacco-and-vapinglegislation n 13153114.html

29. Food and Drugs Act (R.S.C. 1985, c. F27). December 12, 2016. Queen's Printer, Country of Canada. Retrieved from: http://lawslois.justice.gc.ca/PDF/F-27.pdf

30. Norris, S., Tiedemann, M. (2016). An Act to amend the Tobacco Act and the Nonsmokers' Health Act and to make consequential amendments to other Acts. Library of Parliament Publication No. 42-1-S5-E. Retrieved from: http://ectaofcanada.com/legislativesummary-of-bill-s-5/

31. Hess, C. A., Olmedo, P., Navas-Acien, A., Goessier, W., Cohen, J.E., Rule, A.M. (2017). E-cigarettes as a source of toxic and potentially carcinogenic metals. Environmental Research. 152: 221-225

32. Heacock, H., Karakilic, V (2015). Research Method Module 4: Research Design \& Data Collection. Personal Collection of $\mathrm{H}$. Heacock, British Columbia Institute of Technology, Burnaby BC 